\title{
Bioassessment of flaxseed powder and extract against hyperglycemia and hypercholesterolemia using Sprague Dawley rats
}

\author{
Umair Afzal ${ }^{1}$, Masood Sadiq Butt ${ }^{2}$, Faiza Ashfaq ${ }^{3}$, Ahmad Bilal $^{4}$ and Hafiz Ansar Rasul Suleria ${ }^{5,6,7,8^{*}}$ (B)
}

\begin{abstract}
Background: Flaxseed has emerged as a potential source of bioactive components that can be utilized in routine diet to address lifestyle disorders.

Methods: In this context, three studies were carried out on the basis of induction therapies i.e. Study I (Normal diet), Study II (Hyperglycemic diet; 40\% sucrose) and Study III (Hypercholesterolemic diet; 1.5\% cholesterol) using Sprague Dawley rats. Each study was further split into three groups based on diets; Control (free from flaxseed powder or extract), Functional diet (incorporation of flaxseed powder; 10\%) and Nutraceutical diet (inclusion of ethanolic extract of flaxseed; 5\%). During experimental period, hyperglycemic and hyperlipidemic parameters were evaluated alongside, alterations in hematological aspects were also assessed.

Results: Feed intake and body weight demonstrated significant response $(p<0.05)$ of diets and study intervals however, water intake was substantially influenced by study intervals. In study II (hyperglycemic rats), maximum decline in glucose level was recorded (9.02\%) in rats administered with extract based diet. In the same group, maximum increase in insulin (5.90\%) was noted. Regarding lipid profile, the bioevaluation trials revealed maximum reduction in serum cholesterol (13.10\%) in study III (hypercholesterolemic rats) on the provision of flaxseed extract (nutraceutical diet) followed by flaxseed powder (functional diet) i.e. $7.85 \%$. Further, maximum decrease in low density lipoprotein-cholesterol (LDL-c) was reported i.e. $14.28 \%$ on supplementation of flaxseed extract to hypercholesterolemic rats.
\end{abstract}

Conclusions: Thus, flaxseed extract based intervention has shown higher bioefficacy to address hyperglycemia and hypercholesterolemia in comparison to flaxseed powder.

Keywords: Lifestyle disorders, Feed and water intakes, Body weight, Functional diet, Nutraceutical diet

\section{Background}

The trend of consumers towards natural cure through dietary modification is the reason for the immense popularity of nutraceuticals and functional foods. Due to the presence of bioactive moieties, dietary interventions are negatively associated with lifestyle related disorders

\footnotetext{
* Correspondence: hafiz.suleria@uqconnect.edu.au

${ }^{5}$ UQ Diamantina Institute, Translational Research Institute, Faculty of Medicine, The University of Queensland, 37 Kent Street Woolloongabba, Brisbane, QLD 4102, Australia

${ }^{6}$ Centre for Chemistry and Biotechnology, School of Life and Environmental Sciences, Deakin University, Pigdons Road, Waurn Ponds, VIC 3216, Australia Full list of author information is available at the end of the article
}

such as diabetes, hypercholesterolemia, aging and related oxidative stress like conditions [1-4]. Nowadays, diabetes is faced by $4 \%$ of the population throughout the globe and expected to reach up to $5.4 \%$ by 2025 . This chronic metabolic ailment is characterized by high blood glucose level in response to poor insulin release or sensitivity [5]. On the other hand, hypercholesterolemia is a major risk factor of coronary heart diseases i.e. associated with elevated cholesterol, triacylglycerols and LDL-c levels along with suppression of high density lipoprotein-cholesterol (HDL-c) [6]. 
Flaxseed (Linum usitatissimum) is an annual species of the Linaceae family. It is a worthy source of high quality proteins, soluble fiber and polyunsaturated fatty acids [7]. Flaxseed is a rich source of $\alpha$-linolenic acids (57\% of total fat content i.e. $42.16 \mathrm{~g}$ per $100 \mathrm{~g}$ of flaxseed) and dietary fiber i.e. $40 \%$ per $100 \mathrm{~g}$ of flaxseed, in which $30 \%$ is insoluble in nature [8-11]. Besides that, secoisolariciresinol diglucoside (SDG) is a primary lignan in flaxseed, found up to 3236-3699 $\mu \mathrm{g} / \mathrm{g}$ of flaxseed hence attracts scientific fraternity to investigate its health perspectives [12]. SDG molecules linked together to form lignan oligomers also called as lignan macromolecules [13, 14]. Microflora in large intestine deglycosylate lignans after digestion and transformed them into mammalian lignans known as enterodiol (END) and enterolactone (ENL). These metabolites possess high antioxidant activity resultantly suppress mRNA expression of sterol regulatory element binding protein-1c (SREBP-1c), that modulates the activity of cholesterol and fatty acid synthetase enzymes hence likely to control hypercholesterolemia. Further, antioxidant ability of flaxseed protects pancreatic cells and normalizes insulin levels, and reduces the expression of phosphoenolpyruvate carboxykinase gene that aid in controlling diabetes [10, 15]. Traditionally, lignans are recovered through solvent extraction technique, usually with organic solvents like acetone, methanol and ethanol as these phenolic compounds are polar in nature [16].

Keeping in view, the present project was designed to assess the potential of flaxseed powder $(10 \%)$ and flaxseed ethanolic extract $(5 \%)$ to address hyperglycemia and hypercholesterolemia using rodent model trial.

\section{Methods}

\section{Chemicals and preparation of raw materials}

The study was carried out in the Functional and Nutraceutical Food Research Section, National Institute of Food Science and Technology, University of Agriculture Faisalabad (NIFSAT-UAF), Pakistan. Flaxseed seeds were procured from Oil Seed Research Institute, Ayub Agriculture Research Institute, Faisalabad. Flaxseed seeds were cleaned and ground (Renker, Model: GMO 1 grinder) prior experimentation. Reagents and standards were purchased from Merck (Merck KGaA, Darmstadt, Germany) and Sigma-Aldrich (Sigma-Aldrich Tokyo, Japan). Male Sprague Dawley rats used in the efficacy trials were acquired from National Institute of Health (NIH) Islamabad. Diagnostic Kits used were from Sigma-Aldrich, Bioassay (Bioassays Chemical Co. Germany) and Cayman Chemicals (Cayman Europe, Estonia).

\section{Preparation of flaxseed extracts}

Ethanolic extract of flaxseed was prepared through conventional solvent extraction process, conditions include aqueous ethanol $(50 \% \mathrm{v} / \mathrm{v}), 60 \mathrm{~min}$ and $50 \mathrm{C}$ following the methods of Ho et al. [16]. Further, ethanolic extract was filtered and concentrated via rotary evaporator (Eyela, Japan).

\section{Bioefficacy assessment}

For efficacy trials, sixty rats were acquired from National Institute of Health (NIH), Islamabad and kept in Animal Room of NIFSAT-UAF. Initially, some rats were sacrificed to get baseline values for the study. The study was carried out in three categories separately. Study I comprised of rats fed on normal diet, whereas in study II \& III, high glucose \& high cholesterol diets were administered to rats, respectively. During entire experimental period, the Animal Room was maintained at a temperature of $23 \pm 2 \mathrm{C}$ and relative humidity of $55 \pm$ $5 \%$. For an initial period of one week, basal diet was given to the rats to acclimatize them to the environment. Later, normal, functional and nutraceutical diets were administered to the respective groups under each study to evaluate the effects of individual treatment on the selected parameters including serum lipid profile and glucose \& insulin levels. Feed and water intakes were measured daily throughout the experimental period, while body weight was assessed on weekly basis. At termination of animal study, overnight fasted rats were sacrificed. Blood samples of rats were collected through cardiac puncture in non-coated tubes (yellow capped vials) to measure serum lipid profile, glucose \& insulin levels through Microlab-300, Merck, Germany and EDTA coated tubes (purple capped vials) were employed to assess hematological aspects via Medonic M Series; Boule Diagnostics Int AB Stockholm, Sweden.

\section{Study I: Normal diet}

In study I, normal diet was given to the rats to check the effect of functional and nutraceutical diets.

\section{Study II: hyperglycemic diet}

Hyperglycemic diet based on $40 \%$ sucrose was administered to the rats to determine the effect on serum glucose and insulin levels. Simultaneously, the efficacy of functional and nutraceutical diets against hyperglycemic rats was assessed.

\section{Study III: Hypercholesterolemic diet}

Hypercholesterolemic diet containing 1.5\% of cholesterol was fed to rats to alter their lipid profile i.e. cholesterol, HDL-c, LDL-c and triacylglycerol levels. Afterwards, flaxseed powder and extract based diets were provided to the respective rat groups to evaluate hypocholesterolemic potential (Table 1). 
Feed \& water intakes and body weight assessments

Average feed intake of each group was measured on daily basis by eliminating spilt diet from the total diet given during the whole study period [17]. The water intake for each group was also recorded on daily basis. Gain in body weight of experimental groups was measured weekly throughout the study period to monitor the effect of different diets on body weight gain.

\section{Glycemic features}

The collected sera were evaluated for glucose by enzymatic photometric test method GOD-PAP (Breuer \& Breuer Diagnostic, Germany) as described by Kim et al. [18], whereas insulin level was assessed following the method of Ahn et al. [19].

\section{Lipid profile}

Serum cholesterol level of rats was measured by Fluitest Chol (Cholesterin CHOD-PAP) using commercial kit; (Biocon, Vohl-Marienhagen, Germany) by following the protocol of Kim et al. [18]. Triacylglycerols in serum samples was estimated by Fluitest TG (Triglyceride GPO-PAP) kit method as illustrated by Buriro and Tayyab [20]. HDL-c in serum samples were calculated by HDL precipitant method using commercially available Ecolin kits (Merck, Germany) as mentioned by Alshatwi et al. [21]. Further, LDL-c was calculated by using following formula;

$$
\text { LDL-c }=\text { Total cholesterol-HDL-c-VLDL-c }
$$

\section{Hematological aspects}

Total red blood cell (RBC), white blood cell (WBC) and platelets count were determined using Medonic $M$
Series; Boule Diagnostics Int AB Stockholm, Sweden $[22,23]$.

\section{Statistical analysis}

The data for each parameter were subjected to statistical modeling through completely randomized design (CRD) using Statistix 8.1. Furthermore, level of significance was also estimated $(p<0.05)$ using analysis of variance (ANOVA) technique (two-factorial analysis) followed by posthoc Tukey's honestly significant difference (HSD) multiple comparison tests for means separation [24].

\section{Results}

\section{Feed \& water intakes and body weight assessments}

Statistical inference indicated significant effect of diets and study intervals on feed intake in study I (normal rats), study II (hyperglycemic rats) and study III (hypercholesterolemic rats). In study I, maximum feed intake was observed in control diet group $(16.25 \mathrm{~g} / \mathrm{rat} /$ day $)$ followed by nutraceutical $(15.83 \mathrm{~g} / \mathrm{rat} /$ day $)$ and functional (15.73 g/rat/day) groups. During 8-week trial, feed intake in control, functional and nutraceutical groups has increased from 13.77 to $19.66,13.42$ to 18.77 and 13.17 to $18.56 \mathrm{~g} / \mathrm{rat} /$ day, respectively. Means of study II (hyperglycemic rats) portrayed maximum feed intake in control $17.02 \mathrm{~g} / \mathrm{rat} /$ day followed by functional and nutraceutical groups as 16.28 and $15.79 \mathrm{~g} /$ rat/day, respectively. In study II, feed intake increased in control, functional and nutraceutical groups from 1st to 8th week. Study III (hypercholesterolemic rats) demonstrated maximum feed intake $(17.31 \mathrm{~g} / \mathrm{rat} /$ day $)$ in control while minimum level $(16.59 \mathrm{~g} / \mathrm{rat} /$ day $)$ was observed in nutraceutical diet group. Throughout the trial, maximum increment in feed intake was observed in control group from 14.42 to $20.98 \mathrm{~g} / \mathrm{rat} /$ day, functional group from 14.17 to $20.07 \mathrm{~g} /$

Table 1 Diet composition

\begin{tabular}{|c|c|c|c|c|c|c|c|c|c|}
\hline \multirow[t]{2}{*}{ Ingredients (\%) } & \multicolumn{3}{|c|}{ Study I (Normal diet) } & \multicolumn{3}{|c|}{ Study II (Hyperglycemic diet) } & \multicolumn{3}{|c|}{ Study III (Hypercholesterolemic diet) } \\
\hline & Control & Functional diet & Nutraceutical diet & Control & Functional diet & Nutraceutical diet & Control & Functional diet & Nutraceutical diet \\
\hline Corn oil & 10 & 10 & 10 & 10 & 10 & 10 & 10 & 10 & 10 \\
\hline Corn starch & 66 & 56 & 61 & 26 & 16 & 21 & 64.5 & 54.5 & 59.5 \\
\hline Casein & 10 & 10 & 10 & 10 & 10 & 10 & 10 & 10 & 10 \\
\hline Cellulose & 10 & 10 & 10 & 10 & 10 & 10 & 10 & 10 & 10 \\
\hline Mineral mix & 3 & 3 & 3 & 3 & 3 & 3 & 3 & 3 & 3 \\
\hline Vitamin mix & 1 & 1 & 1 & 1 & 1 & 1 & 1 & 1 & 1 \\
\hline Sucrose & - & - & - & 40 & 40 & 40 & - & - & - \\
\hline Cholesterol & - & - & - & - & - & - & 1.5 & 1.5 & 1.5 \\
\hline Flaxseed powder & - & 10 & - & - & 10 & - & - & 10 & - \\
\hline Flaxseed extract & - & - & 5 & - & - & 5 & - & - & 5 \\
\hline
\end{tabular}

Mineral mix (per $100 \mathrm{~g}$ D.W.) calcium: $7.8 \mathrm{~g}$, phosphorus: $7.6 \mathrm{~g}$, potassium: $6.9 \mathrm{~g}$, sulfur: $1.8 \mathrm{~g}$, magnesium: $1.5 \mathrm{~g}$, sodium: $1.5 \mathrm{~g}$, choline: $0.35 \mathrm{~g}$, iron: $190 \mathrm{mg}$, manganese: $90 \mathrm{mg}$, zinc: $54 \mathrm{mg}$, copper: $7 \mathrm{mg}$, selenium: $4 \mathrm{mg}$, cobalt: $4 \mathrm{mg}$ and iodine: $1 \mathrm{mg}$

Vitamin mix (per 100 g D.W.) cholecalciferol: $2000 \mathrm{IU}$, vitamin A palmitate: $1442 \mathrm{IU}$, niacin: $7.7 \mathrm{mg}$, folacin: $5.4 \mathrm{mg}$, a-tocopherol acetate: $4.1 \mathrm{mg}$, pyridoxine: 1.9 mg, thiamin: $1.2 \mathrm{mg}$, vitamin $B_{12}: 1.0 \mathrm{mg}$, vitamin $B_{3}: 0.9 \mathrm{mg}$, riboflavin: $0.4 \mathrm{mg}$ and vitamin $\mathrm{K}_{3}: 0.1 \mathrm{mg}$ 
rat/day and nutraceutical group from 14.03 to $19.91 \mathrm{~g} /$ rat/day (Fig. 1).

Statistical analysis explicated non-substantial response of diets however, study intervals presented significant impact on water intake in study I (normal rats), study II (hyperglycemic rats) and study III (hypercholesterolemic rats). In study I, maximum water intake was viewed in nutraceutical group $(22.44 \mathrm{~mL} / \mathrm{rat} /$ day $)$ followed by functional group $(22.25 \mathrm{~mL} / \mathrm{rat} /$ day $)$ and control $(\mathrm{mL} / \mathrm{rat} /$ day $\mathrm{mg} / \mathrm{dL})$.
Throughout the experimentation phase, water intake in control, functional and nutraceutical groups raised from 18.16 to $25.73,18.38$ to 25.95 and 18.54 to $26.11 \mathrm{~mL} / \mathrm{rat} /$ day, respectively. Means of study II (hyperglycemic rats) indicated maximum water intake in nutraceutical group $23.48 \mathrm{~mL} / \mathrm{rat} /$ day followed by functional group $23.28 \mathrm{~mL} /$ rat/day and control group $23.04 \mathrm{~mL} /$ rat/day. In study II, water intake increased in control group from 19.16 to $27.27 \mathrm{~mL} / \mathrm{rat} /$ day and functional and nutraceutical groups

a

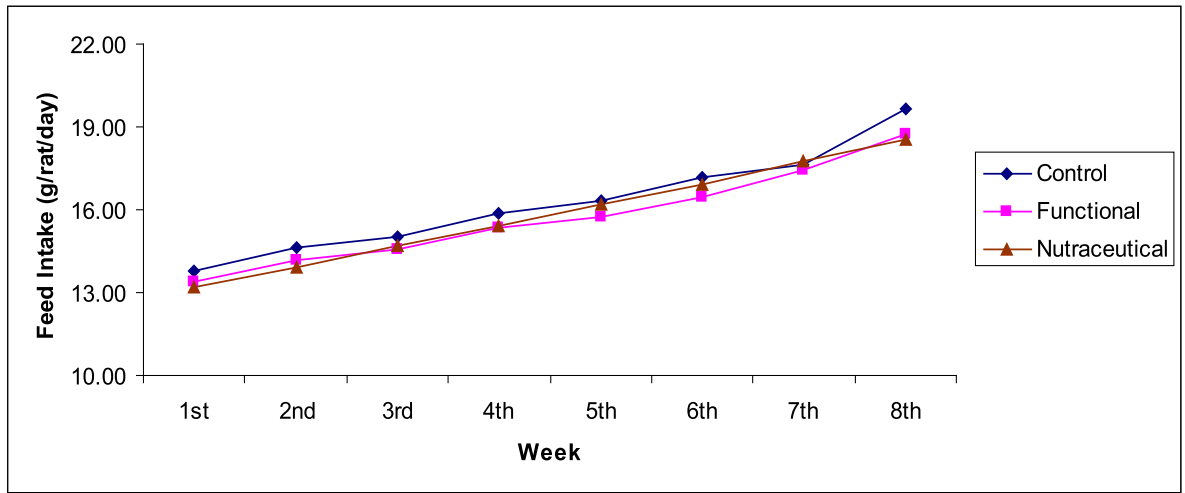

b

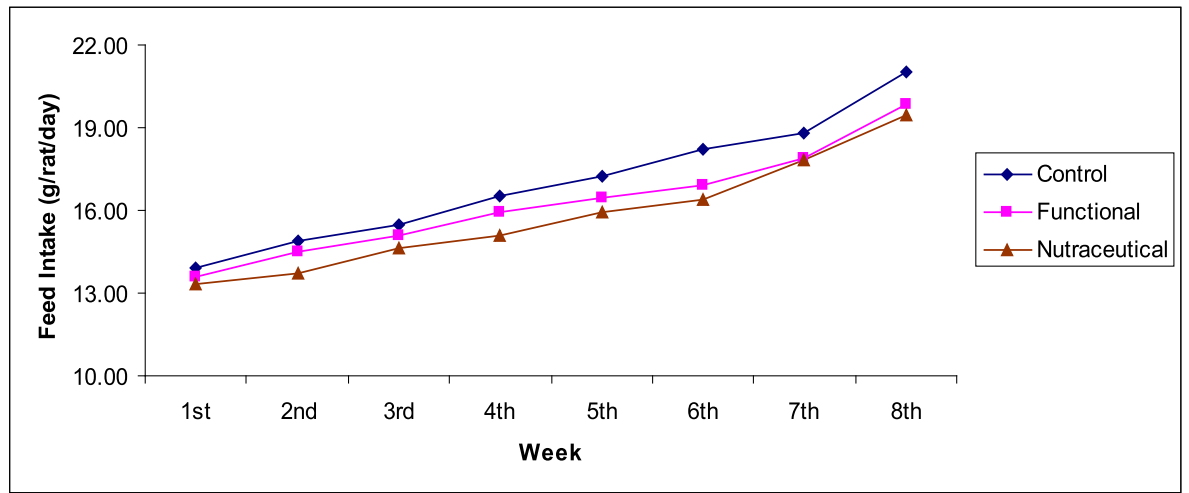

c

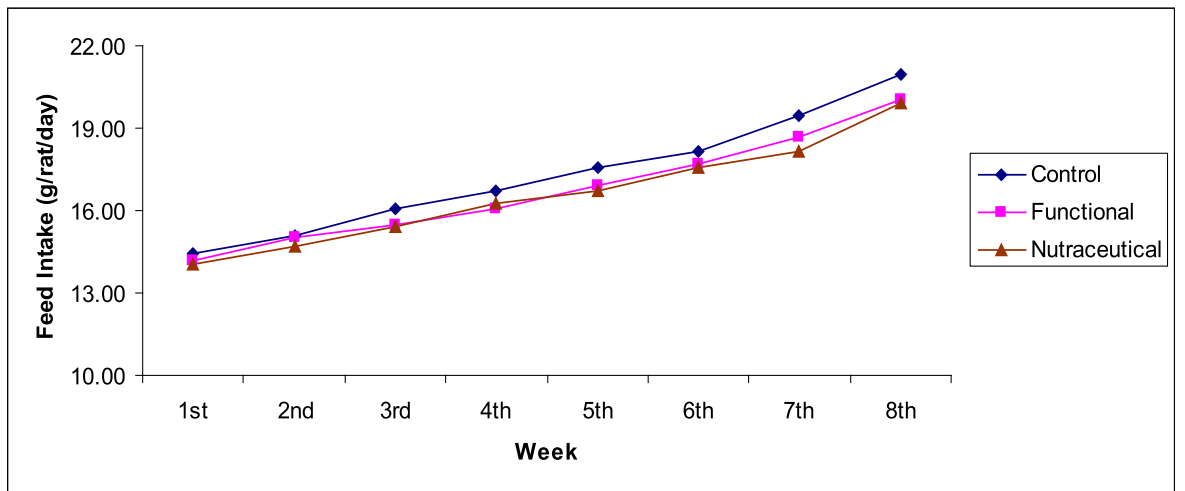

Fig. 1 Feed intake in different studies (g/rat/day) (a) Study I: Normal rats; (b) Study II: Hyperglycemic rats; and (c) Study III: Hypercholesterolemic rats 
a

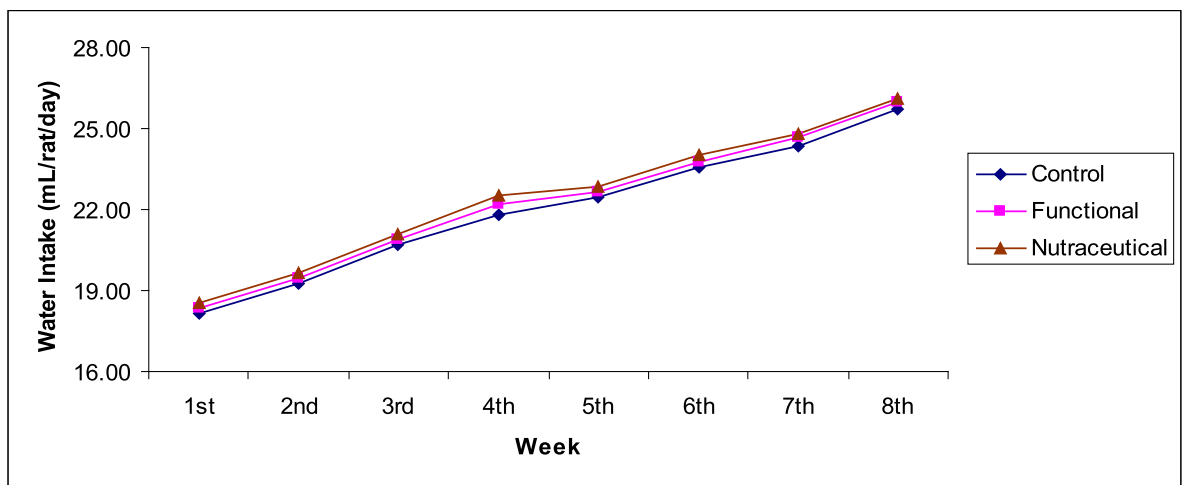

b

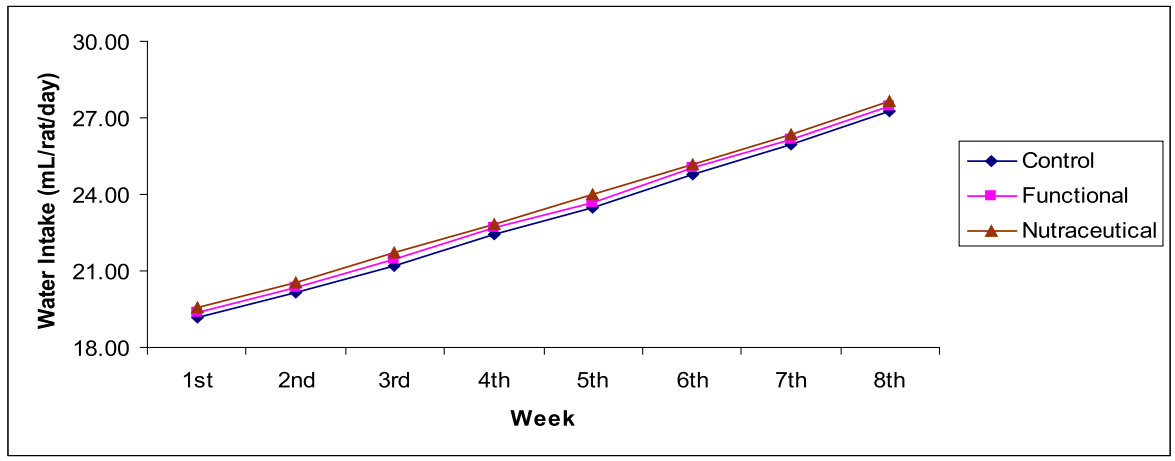

c

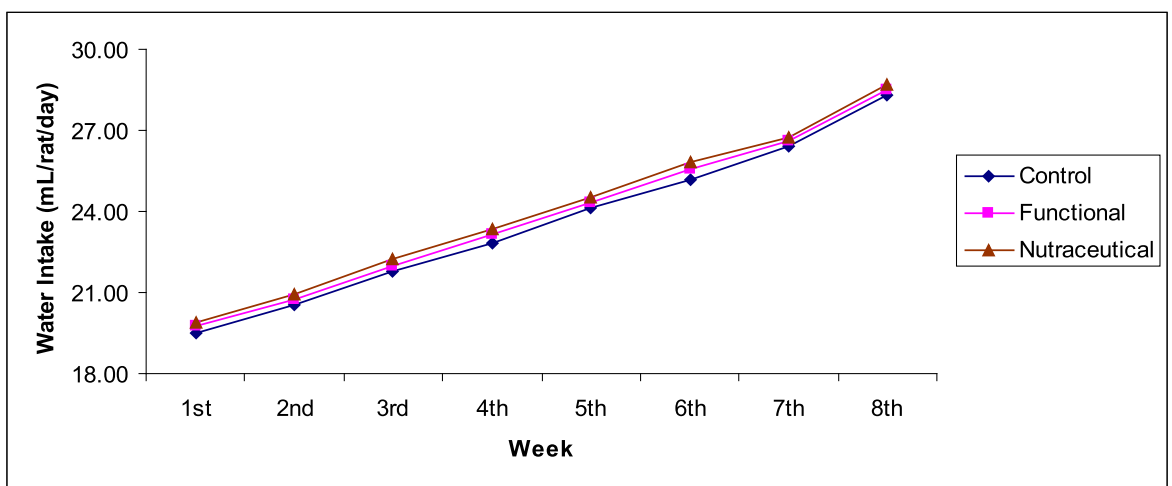

Fig. 2 Water intake in different studies (mL/rat/day) (a) Study I: Normal rats; (b) Study II: Hyperglycemic rats; and (c) Study III: Hypercholesterolemic rats

also showed significant increment in water intake. Further, study III (hypercholesterolemic rats) depicted maximum water intake $(24.03 \mathrm{~mL} / \mathrm{rat} /$ day $)$ in nutraceutical group however, water intake up to 23.83 and $23.58 \mathrm{~mL} /$ rat/day were noticed in functional and control groups, accordingly. During animal study period, maximum increase was observed in control group from 19.52 to $28.29 \mathrm{~mL} /$ rat/day. Moreover, water intake improved from 19.74 to 28.51 and 19.90 to $28.67 \mathrm{~mL} /$ rat/day in functional and nutraceutical groups, respectively (Fig. 2).
Statistical interpretation revealed significant effect of diets and experiment intervals on body weight in study I (normal rats), study II (hyperglycemic rats) and study III (hypercholesterolemic rats). In study I, maximum body weight was observed in control $(174.59 \mathrm{~g})$ followed by functional $(167.64 \mathrm{~g})$ and nutraceutical $(163.56 \mathrm{~g})$ groups. During 8-week trial, body weight in control, functional and nutraceutical groups had raised from 132.02 to $223.50 \mathrm{~g}, 128.69$ to $212.09 \mathrm{~g}$ and 125.75 to $207.86 \mathrm{~g}$, respectively. Means of study II (hyperglycemic rats) had 
a

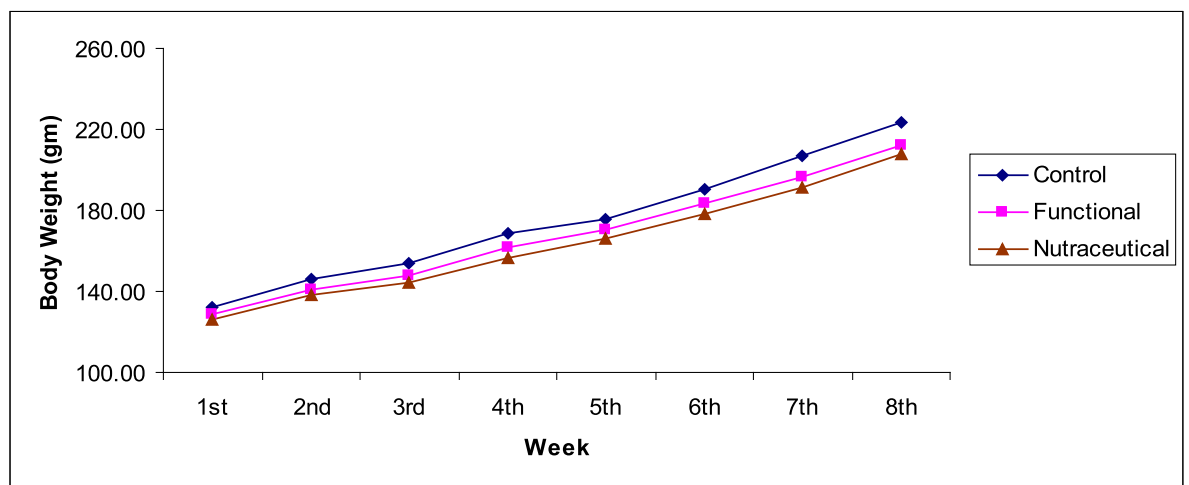

b

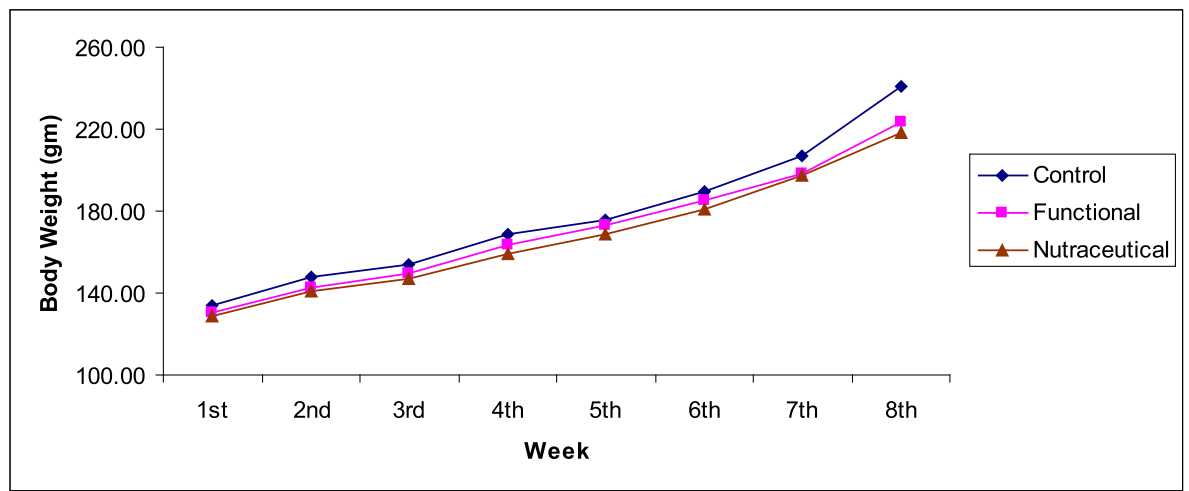

c

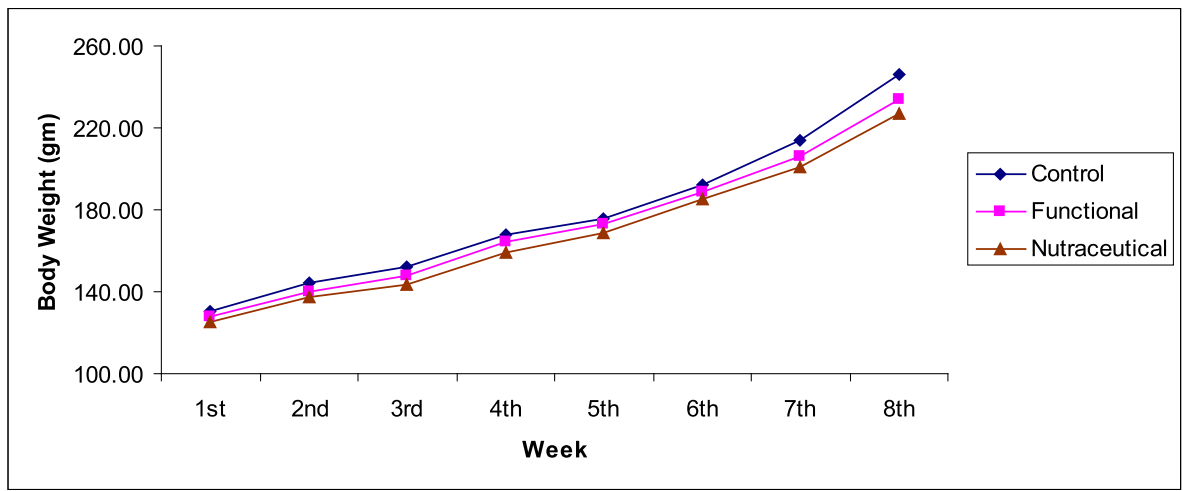

Fig. 3 Body weight of rats in different studies (g/rat) (a) Study I: Normal rats; (b) Study II: Hyperglycemic rats; and (c) Study III: Hypercholesterolemic rats

shown maximum body weight in control $177.17 \mathrm{~g}$ followed by functional and nutraceutical groups as 170.89 and $167.62 \mathrm{~g}$, respectively. In study II, maximum body weight was recorded in control group as 240.67 from $134.31 \mathrm{~g}$. Further, significant increase in body weight was also found in functional and nutraceutical groups. Study III (hypercholesterolemic rats) showed maximum body weight $(177.74 \mathrm{~g})$ in control while minimum weight gain was noted in nutraceutical group
(172.83 g). During 8-week trial, maximum increase was observed in control group from 130.04 to $246.42 \mathrm{~g}$ and functional and nutraceutical groups also showed increase in body weight from 128.18 to $234.20 \mathrm{~g}$ and 125.04 to 227.12 g, respectively (Fig. 3).

\section{Glycemic features}

Statistical scrutiny presented non-substantial effect of diets and study intervals on glucose level in study I 
Table 2 Effect of diets and study intervals on glucose level $(\mathrm{mg} / \mathrm{dL})$ of rats in different studies

\begin{tabular}{|c|c|c|c|c|c|}
\hline \multirow[t]{2}{*}{ Studies } & \multirow[t]{2}{*}{ Diets } & \multicolumn{3}{|c|}{ Study intervals (Days) } & \multirow[t]{2}{*}{ Means } \\
\hline & & 0 & 28 & 56 & \\
\hline \multirow[t]{4}{*}{ Study 1} & Control & $89.72 \pm 4.48$ & $89.95 \pm 4.49$ & $90.85 \pm 3.63$ & $90.17 \pm 5.12$ \\
\hline & Functional & $90.05 \pm 4.62$ & $89.02 \pm 3.82$ & $88.62 \pm 3.32$ & $89.91 \pm 4.94$ \\
\hline & Nutraceutical & $91.46 \pm 4.81$ & $89.56 \pm 4.32$ & $88.73 \pm 3.72$ & $89.23 \pm 4.12$ \\
\hline & Means & $90.41 \pm 4.78$ & $89.51 \pm 4.72$ & $89.40 \pm 4.54$ & \\
\hline \multirow[t]{4}{*}{ Study II } & Control & $122.12 \pm 6.10^{b}$ & $135.47 \pm 6.77^{\mathrm{a}}$ & $139.35 \pm 6.96^{\mathrm{a}}$ & $132.31 \pm 6.42^{a}$ \\
\hline & Functional & $118.35 \pm 5.91^{\mathrm{bcd}}$ & $113.17 \pm 5.40^{\text {cde }}$ & $109.78 \pm 5.26^{\mathrm{e}}$ & $113.77 \pm 5.08^{b}$ \\
\hline & Nutraceutical & $117.08 \pm 5.72^{\mathrm{bc}}$ & $109.15 \pm 5.12^{\mathrm{de}}$ & $106.51 \pm 4.68^{\mathrm{e}}$ & $110.91 \pm 5.42^{b}$ \\
\hline & Means & $119.18 \pm 5.66$ & $119.26 \pm 5.37$ & $118.54 \pm 5.06$ & \\
\hline \multirow[t]{4}{*}{ Study 111} & Control & $95.69 \pm 5.22^{b c}$ & $101.95 \pm 5.56^{\mathrm{ab}}$ & $104.73 \pm 6.12^{a}$ & $100.79 \pm 5.42^{\mathrm{a}}$ \\
\hline & Functional & $97.14 \pm 5.82^{\mathrm{abc}}$ & $95.81 \pm 5.44^{\mathrm{bc}}$ & $94.17 \pm 5.08^{b c}$ & $95.71 \pm 5.06^{b}$ \\
\hline & Nutraceutical & $96.55 \pm 6.24^{\mathrm{abc}}$ & $93.19 \pm 5.32^{c}$ & $91.86 \pm 4.82^{c}$ & $93.87 \pm 4.92^{\mathrm{b}}$ \\
\hline & Means & $96.46 \pm 4.8$ & $96.98 \pm 4.65$ & $96.92 \pm 4.32$ & \\
\hline
\end{tabular}

Study I (Normal rats), Study II (Hyperglycemic rats), Study III (Hypercholesterolemic rats). Lettering indicates significant differences between means using ANOVA (two-way factorial) with posthoc Tukey's honestly significant difference (HSD) test

(normal rats). The effect of diets was significant however, response of study intervals was non-significant in study II (hyperglycemic rats) and study III (hypercholesterolemic rats). The decrease in glucose level of normal rats fed on functional and nutraceutical diets were 1.57 and $2.98 \%$, respectively. In glucose level, $7.21 \%$ reduction was observed in hyperglycemic rats fed on functional diet while $9.02 \%$ decrease was noted via nutraceutical diet. Hypercholesterolemic rats fed on functional and nutraceutical diets resulted in decrement in glucose up to 3.05 and $4.85 \%$, accordingly (Table 2 ).

Regarding insulin level, non-significant effect of diets and study intervals was noted in study I (normal rats). However, significant effect of diets and nonsignificant effect of study intervals was viewed with respect to insulin level in study II (hyperglycemic rats) and study III (hypercholesterolemic rats). The insulin levels of normal rats fed on functional and nutraceutical diets were 1.06 and $1.93 \%$, respectively. The increase in insulin level (4.46\%) was observed in hyperglycemic rats fed on functional diet while it increased up to $5.89 \%$ in hyperglycemic rats administered with nutraceutical diet. Insulin level of hypercholesterolemic rats fed on functional and nutraceutical diets increased by 2.22 and $3.87 \%$, respectively (Table 3 ).

Table 3 Effect of diets and study intervals on insulin level $(\mu \mathrm{U} / \mathrm{mL})$ of rats in different studies

\begin{tabular}{|c|c|c|c|c|c|}
\hline \multirow[t]{2}{*}{ Studies } & \multirow[t]{2}{*}{ Diets } & \multicolumn{3}{|c|}{ Study intervals (Days) } & \multirow[t]{2}{*}{ Means } \\
\hline & & $\overline{0}$ & 28 & 56 & \\
\hline \multirow[t]{4}{*}{ Study 1} & Control & $9.17 \pm 0.32$ & $9.22 \pm 0.38$ & $9.26 \pm 0.42$ & $9.21 \pm 0.41$ \\
\hline & Functional & $9.49 \pm 0.51$ & $9.56 \pm 0.58$ & $9.59 \pm 0.54$ & $9.54 \pm 0.55$ \\
\hline & Nutraceutical & $9.57 \pm 0.47$ & $9.69 \pm 0.54$ & $9.75 \pm 0.48$ & $9.67 \pm 0.46$ \\
\hline & Means & $9.41 \pm 0.42$ & $9.47 \pm 0.48$ & $9.53 \pm 0.49$ & \\
\hline \multirow[t]{4}{*}{ Study ॥ } & Control & $10.08 \pm 0.50$ & $10.14 \pm 0.61$ & $10.21 \pm 0.57$ & $10.14 \pm 0.54^{b}$ \\
\hline & Functional & $10.34 \pm 0.52$ & $10.71 \pm 0.56$ & $10.85 \pm 0.60$ & $10.63 \pm 0.58^{\mathrm{ab}}$ \\
\hline & Nutraceutical & $10.66 \pm 0.58$ & $11.05 \pm 0.54$ & $11.29 \pm 0.63$ & $11.00 \pm 0.62^{\mathrm{a}}$ \\
\hline & Means & $10.36 \pm 0.54$ & $10.56 \pm 0.58$ & $10.65 \pm 0.68$ & \\
\hline \multirow[t]{4}{*}{ Study 111} & Control & $9.36 \pm 0.35$ & $9.41 \pm 0.39$ & $9.48 \pm 0.44$ & $9.42 \pm 0.58$ \\
\hline & Functional & $9.68 \pm 0.54$ & $9.83 \pm 0.46$ & $9.89 \pm 0.37$ & $9.80 \pm 0.43$ \\
\hline & Nutraceutical & $9.86 \pm 0.49$ & $10.12 \pm 0.54$ & $10.24 \pm 0.42$ & $10.07 \pm 0.41$ \\
\hline & Means & $9.63 \pm 0.44$ & $9.78 \pm 0.48$ & $9.87 \pm 0.44$ & \\
\hline
\end{tabular}

Study I (Normal rats), Study II (Hyperglycemic rats), Study III (Hypercholesterolemic rats). Lettering indicates significant differences between means using ANOVA (two-way factorial) with posthoc Tukey's honestly significant difference (HSD) test 
Table 4 Effect of diets and study intervals on cholesterol $(\mathrm{mg} / \mathrm{dL})$ of rats in different studies

\begin{tabular}{|c|c|c|c|c|c|}
\hline \multirow[t]{2}{*}{ Studies } & \multirow[t]{2}{*}{ Diets } & \multicolumn{3}{|c|}{ Study intervals (Days) } & \multirow[t]{2}{*}{ Means } \\
\hline & & 0 & 28 & 56 & \\
\hline \multirow[t]{4}{*}{ Study 1} & Control & $79.95 \pm 3.19$ & $80.70 \pm 4.86$ & $81.11 \pm 2.43$ & $80.58 \pm 4.65$ \\
\hline & Functional & $80.47 \pm 3.21$ & $79.59 \pm 3.05$ & $79.30 \pm 5.30$ & $79.78 \pm 3.84$ \\
\hline & Nutraceutical & $78.52 \pm 4.71$ & $77.28 \pm 4.68$ & $76.75 \pm 5.32$ & $77.51 \pm 3.91$ \\
\hline & Means & $79.65 \pm 4.77$ & $79.19 \pm 3.14$ & $79.05 \pm 4.43$ & \\
\hline \multirow[t]{4}{*}{ Study $\|$} & Control & $109.26 \pm 6.55$ & $115.82 \pm 4.63$ & $119.08 \pm 5.95$ & $114.72 \pm 4.58^{\mathrm{a}}$ \\
\hline & Functional & $108.50 \pm 5.51$ & $105.54 \pm 4.06$ & $103.91 \pm 4.94$ & $105.98 \pm 5.15^{b}$ \\
\hline & Nutraceutical & $108.82 \pm 5.61$ & $103.28 \pm 4.96$ & $101.56 \pm 3.85$ & $104.55 \pm 5.07^{b}$ \\
\hline & Means & $108.86 \pm 4.35$ & $108.21 \pm 3.16$ & $108.18 \pm 5.24$ & \\
\hline \multirow[t]{4}{*}{ Study 111} & Control & $128.69 \pm 5.14^{c}$ & $140.43 \pm 8.42^{b}$ & $148.02 \pm 4.40^{\mathrm{a}}$ & $139.05 \pm 4.17^{\mathrm{a}}$ \\
\hline & Functional & $128.06 \pm 5.12^{\mathrm{cd}}$ & $121.55 \pm 3.49^{\mathrm{de}}$ & $118.01 \pm 5.76^{\mathrm{e}}$ & $122.54 \pm 5.95^{b}$ \\
\hline & Nutraceutical & $127.31 \pm 5.45^{\mathrm{cd}}$ & $115.98 \pm 4.55^{\mathrm{ef}}$ & $110.63 \pm 5.47^{f}$ & $117.97 \pm 6.01^{c}$ \\
\hline & Means & $128.02 \pm 6.40$ & $125.99 \pm 4.94$ & $125.55 \pm 5.40$ & \\
\hline
\end{tabular}

Study I (Normal rats), Study II (Hyperglycemic rats), Study III (Hypercholesterolemic rats). Lettering indicates significant differences between means using ANOVA (two-way factorial) with posthoc Tukey's honestly significant difference (HSD) test

\section{Lipid profile}

The effect of diets and study intervals was significant on serum cholesterol in study I (normal rats). In study II (hyperglycemic rats) and study III (hypercholesterolemic rats), serum cholesterol was significantly affected by diets while non-significantly with respect to study duration. The serum cholesterol of normal rats fed on functional diet was decreased up to $1.45 \%$, whereas $2.25 \%$ reduction was observed on supplementation of nutraceutical diet. A reduction of $4.23 \%$ was observed in serum cholesterol level of hyperglycemic rats + functional diet group while it reduced up to $6.65 \%$ in hyperglycemic rats fed on nutraceutical diet. Serum cholesterol of hypercholesterolemic rats provided with functional and nutraceutical diets were decreased by 7.84 and $13.10 \%$, respectively (Table 4 ).

Statistical analysis portrayed significant impact of diets on triacylglycerol level however, non-substantial effect of study intervals was noticed on triacylglycerol level in study I (normal rats). Further, the effect of diets was significant while impact of study intervals was nonsignificant in study II (hyperglycemic rats) and study III (hypercholesterolemic rats). The decrease in triacylglycerol level of normal rats was $3.27 \%$ fed on functional diet, whereas $4.85 \%$ reduction was observed in normal rats administered with nutraceutical diet. In hyperglycemic rats, triacylglycerol level was reduced by $6.43 \%$ (functional diet) and $8.76 \%$ (nutraceutical diet).

Table 5 Effect of diets and study intervals on triacylglycerol level $(\mathrm{mg} / \mathrm{dL})$ of rats in different studies

\begin{tabular}{|c|c|c|c|c|c|}
\hline \multirow[t]{2}{*}{ Studies } & \multirow[t]{2}{*}{ Diets } & \multicolumn{3}{|c|}{ Study intervals (Days) } & \multirow[t]{2}{*}{ Means } \\
\hline & & $\overline{0}$ & 28 & 56 & \\
\hline \multirow[t]{4}{*}{ Study 1} & Control & $70.82 \pm 3.52$ & $72.02 \pm 2.88$ & $72.89 \pm 4.02$ & $71.91 \pm 3.59^{a}$ \\
\hline & Functional & $71.61 \pm 2.86$ & $70.253 \pm 4.21$ & $69.26 \pm 3.14$ & $70.37 \pm 3.17^{\mathrm{ab}}$ \\
\hline & Nutraceutical & $70.67 \pm 4.24$ & $68.82 \pm 3.44$ & $67.19 \pm 2.72$ & $68.89 \pm 4.15^{b}$ \\
\hline & Means & $71.03 \pm 3.61$ & $70.36 \pm 3.16$ & $69.78 \pm 4.21$ & \\
\hline \multirow[t]{4}{*}{ Study II } & Control & $75.61 \pm 3.78^{b c}$ & $80.39 \pm 4.01^{\mathrm{a}}$ & $81.98 \pm 4.10^{\mathrm{a}}$ & $79.32 \pm 3.96^{a}$ \\
\hline & Functional & $75.04 \pm 3.02^{b c}$ & $71.84 \pm 3.54^{\mathrm{cd}}$ & $70.21 \pm 3.81^{d}$ & $72.36 \pm 3.58^{b}$ \\
\hline & Nutraceutical & $74.29 \pm 4.12^{\mathrm{b}}$ & $69.41 \pm 3.94^{d}$ & $67.78 \pm 4.42^{d}$ & $70.49 \pm 3.69^{b}$ \\
\hline & Means & $74.98 \pm 4.32$ & $73.88 \pm 3.97$ & $73.32 \pm 3.62$ & \\
\hline \multirow[t]{4}{*}{ Study 111} & Control & $91.24 \pm 4.98^{\mathrm{bc}}$ & $99.52 \pm 5.47^{a}$ & $102.32 \pm 5.11^{\mathrm{a}}$ & $97.69 \pm 4.88^{\mathrm{a}}$ \\
\hline & Functional & $93.75 \pm 4.56^{\mathrm{b}}$ & $87.44 \pm 5.12^{c d}$ & $85.71 \pm 3.82^{d}$ & $88.96 \pm 4.32^{b}$ \\
\hline & Nutraceutical & $92.66 \pm 4.08^{b}$ & $85.69 \pm 4.98^{d}$ & $83.25 \pm 3.67^{d}$ & $87.20 \pm 4.12^{b}$ \\
\hline & Means & $92.55 \pm 4.72$ & $90.88 \pm 5.32$ & $90.42 \pm 4.16$ & \\
\hline
\end{tabular}

Study I (Normal rats), Study II (Hyperglycemic rats), Study III (Hypercholesterolemic rats). Lettering indicates significant differences between means using ANOVA (two-way factorial) with posthoc Tukey's honestly significant difference (HSD) test 
Table 6 Effect of diets and study intervals on LDL-c (mg/dL) of rats in different studies

\begin{tabular}{|c|c|c|c|c|c|}
\hline \multirow[t]{2}{*}{ Studies } & \multirow[t]{2}{*}{ Diets } & \multicolumn{3}{|c|}{ Study intervals (Days) } & \multirow[t]{2}{*}{ Means } \\
\hline & & 0 & 28 & 56 & \\
\hline \multirow[t]{4}{*}{ Study 1} & Control & $31.88 \pm 2.09$ & $32.22 \pm 1.51$ & $32.33 \pm 1.81$ & $32.14 \pm 1.50^{\mathrm{a}}$ \\
\hline & Functional & $31.63 \pm 1.58$ & $30.98 \pm 2.16$ & $30.70 \pm 1.23$ & $31.10 \pm 1.55^{b}$ \\
\hline & Nutraceutical & $31.76 \pm 1.27$ & $30.68 \pm 1.53$ & $30.33 \pm 2.12$ & $30.92 \pm 1.54^{b}$ \\
\hline & Means & $31.75 \pm 1.86$ & $31.29 \pm 1.22$ & $31.12 \pm 1.52$ & \\
\hline \multirow[t]{4}{*}{ Study \| } & Control & $54.87 \pm 3.84^{\mathrm{bcd}}$ & $58.97 \pm 2.94^{\mathrm{ab}}$ & $60.34 \pm 2.41^{a}$ & $58.06 \pm 2.90^{\mathrm{a}}$ \\
\hline & Functional & $56.90 \pm 2.84^{\mathrm{abc}}$ & $53.78 \pm 2.09^{c d}$ & $52.67 \pm 3.56^{c d}$ & $54.45 \pm 2.66^{\mathrm{b}}$ \\
\hline & Nutraceutical & $56.13 \pm 3.36^{\mathrm{abc}}$ & $52.40 \pm 2.52^{\mathrm{cd}}$ & $50.44 \pm 1.94^{d}$ & $52.99 \pm 2.58^{b}$ \\
\hline & Means & $55.96 \pm 3.35$ & $55.05 \pm 2.15$ & $54.48 \pm 2.66$ & \\
\hline \multirow[t]{4}{*}{ Study 111} & Control & $62.72 \pm 3.76^{b}$ & $69.15 \pm 2.76^{a}$ & $71.32 \pm 3.56^{\mathrm{a}}$ & $67.73 \pm 4.74^{a}$ \\
\hline & Functional & $63.92 \pm 3.19^{b}$ & $58.43 \pm 3.44^{c}$ & $56.81 \pm 3.86^{c}$ & $59.72 \pm 3.53^{b}$ \\
\hline & Nutraceutical & $62.20 \pm 2.48^{b}$ & $55.27 \pm 3.25^{c d}$ & $53.32 \pm 2.58^{d}$ & $56.93 \pm 3.92^{c}$ \\
\hline & Means & $62.94 \pm 3.14^{\mathrm{a}}$ & $60.95 \pm 3.61^{b}$ & $60.48 \pm 2.97^{b}$ & \\
\hline
\end{tabular}

Study I (Normal rats), Study II (Hyperglycemic rats), Study III (Hypercholesterolemic rats). Lettering indicates significant differences between means using ANOVA (two-way factorial) with posthoc Tukey's honestly significant difference (HSD) test

Triacylglycerol level of hypercholesterolemic rats fed with functional and nutraceutical diet showed decrease up to 8.57 and $10.14 \%$, respectively (Table 5).

Statistical inference portrayed significant effect of diets on LDL-c while the effect of study intervals was nonsubstantial for study I (normal rats). However, in study II (hyperglycemic rats), diets impacted significantly on LDL-c while non-significant effect was noticed regarding study intervals. In study III (hypercholesterolemic rats), both diets and study intervals impacted substantially on LDL-c. In normal rats, there was a decrease of $2.94 \%$ in LDL-c of rats fed on functional diet and $4.53 \%$ through nutraceutical diet. In hyperglycemic rats, the reductions in LDL-c were up to $7.43 \%$ (functional diet) and $10.18 \%$ (nutraceutical diet) during the whole study period. LDLc of hypercholesterolemic rats fed with functional and nutraceutical diets showed decrease up to 11.11 and $14.28 \%$, respectively (Table 6 ).

Non-substantial response of diets and study intervals was viewed on HDL-c in study I (normal rats) study II (hyperglycemic rats) and study III (hypercholesterolemic rats). The normal rats fed on functional diet responded $1.23 \%$ increase in HDL-c, whereas $1.77 \%$ increment was observed via nutraceutical diet. An increase of $1.21 \%$ was observed in HDL-c of hyperglycemic rats on administration of functional diet while it increased up to $2.03 \%$

Table 7 Effect of diets and study intervals on $\mathrm{HDL}-\mathrm{c}(\mathrm{mg} / \mathrm{dL})$ of rats in different studies

\begin{tabular}{|c|c|c|c|c|c|}
\hline \multirow[t]{2}{*}{ Studies } & \multirow[t]{2}{*}{ Diets } & \multicolumn{3}{|c|}{ Study intervals (Days) } & \multirow[t]{2}{*}{ Means } \\
\hline & & 0 & 28 & 56 & \\
\hline \multirow[t]{4}{*}{ Study 1} & Control & $33.75 \pm 2.02$ & $33.29 \pm 1.33$ & $33.25 \pm 1.66$ & $33.43 \pm 1.02$ \\
\hline & Functional & $33.87 \pm 1.69$ & $34.08 \pm 1.36$ & $34.29 \pm 2.40$ & $34.08 \pm 1.70$ \\
\hline & Nutraceutical & $32.81 \pm 1.96$ & $33.22 \pm 1.33$ & $33.39 \pm 1.67$ & $33.14 \pm 2.32$ \\
\hline & Means & $33.47 \pm 1.67$ & $33.53 \pm 1.34$ & $33.64 \pm 2.36$ & \\
\hline \multirow[t]{4}{*}{ Study II } & Control & $39.05 \pm 2.73$ & $37.68 \pm 2.11$ & $37.22 \pm 2.16$ & $37.98 \pm 1.66$ \\
\hline & Functional & $38.51 \pm 1.92$ & $38.82 \pm 2.43$ & $38.97 \pm 1.63$ & $38.76 \pm 2.82$ \\
\hline & Nutraceutical & $39.84 \pm 1.99$ & $40.38 \pm 2.36$ & $40.64 \pm 1.98$ & $40.28 \pm 2.74$ \\
\hline & Means & $39.13 \pm 1.95$ & $38.96 \pm 2.85$ & $38.94 \pm 1.65$ & \\
\hline \multirow[t]{4}{*}{ Study 111} & Control & $45.12 \pm 2.25$ & $44.53 \pm 3.17$ & $43.79 \pm 2.23$ & $44.48 \pm 3.07$ \\
\hline & Functional & $45.94 \pm 3.21$ & $46.58 \pm 2.39$ & $46.82 \pm 3.38$ & $46.44 \pm 1.42$ \\
\hline & Nutraceutical & $46.71 \pm 2.80$ & $47.68 \pm 2.84$ & $48.02 \pm 2.39$ & $47.48 \pm 2.35$ \\
\hline & Means & $45.92 \pm 3.21$ & $46.26 \pm 2.46$ & $46.21 \pm 2.22$ & \\
\hline
\end{tabular}

Study I (Normal rats), Study II (Hyperglycemic rats), Study III (Hypercholesterolemic rats). Lettering indicates significant differences between means using ANOVA (two-way factorial) with posthoc Tukey's honestly significant difference (HSD) test 
Table 8 Effect of diets and study intervals on RBC $\left(10^{6} / \mu \mathrm{L}\right)$ of rats in different studies

\begin{tabular}{|c|c|c|c|c|c|}
\hline \multirow[t]{2}{*}{ Studies } & \multirow[t]{2}{*}{ Diets } & \multicolumn{3}{|c|}{ Study intervals (Days) } & \multirow[t]{2}{*}{ Means } \\
\hline & & 0 & 28 & 56 & \\
\hline \multirow[t]{4}{*}{ Study 1} & Control & $7.74 \pm 0.16$ & $7.53 \pm 0.25$ & $7.93 \pm 0.20$ & $7.73 \pm 0.21$ \\
\hline & Functional & $7.65 \pm 0.19$ & $7.70 \pm 0.30$ & $7.96 \pm 0.33$ & $7.77 \pm 0.27$ \\
\hline & Nutraceutical & $7.70 \pm 0.15$ & $7.94 \pm 0.16$ & $8.21 \pm 0.19$ & $7.95 \pm 0.17$ \\
\hline & Means & $7.70 \pm 0.12^{b}$ & $7.72 \pm 0.27^{b}$ & $8.03 \pm 0.23^{\mathrm{a}}$ & \\
\hline \multirow[t]{4}{*}{ Study \| } & Control & $8.44 \pm 0.22$ & $8.37 \pm 0.23$ & $8.32 \pm 0.20$ & $8.37 \pm 0.21^{a}$ \\
\hline & Functional & $8.29 \pm 0.23$ & $8.60 \pm 0.28$ & $8.95 \pm 0.26$ & $8.61 \pm 0.24^{b}$ \\
\hline & Nutraceutical & $8.38 \pm 0.22$ & $8.68 \pm 0.25$ & $8.91 \pm 0.23$ & $8.65 \pm 0.22^{b}$ \\
\hline & Means & $8.37 \pm 0.24$ & $8.55 \pm 0.26$ & $8.72 \pm 0.21$ & \\
\hline \multirow[t]{4}{*}{ Study 111} & Control & $9.21 \pm 0.11$ & $9.16 \pm 0.18$ & $9.11 \pm 0.22$ & $9.16 \pm 0.17^{a}$ \\
\hline & Functional & $9.36 \pm 0.19$ & $9.47 \pm 0.16$ & $9.58 \pm 0.21$ & $9.46 \pm 0.18^{b}$ \\
\hline & Nutraceutical & $9.54 \pm 0.14$ & $9.61 \pm 0.21$ & $9.63 \pm 0.19$ & $9.59 \pm 0.20^{b}$ \\
\hline & Means & $9.37 \pm 0.16$ & $9.41 \pm 0.20$ & $9.44 \pm 0.19$ & \\
\hline
\end{tabular}

Study I (Normal rats), Study II (Hyperglycemic rats), Study III (Hypercholesterolemic rats). Lettering indicates significant differences between means using ANOVA (two-way factorial) with posthoc Tukey's honestly significant difference (HSD) test

in hyperglycemic rats fed on nutraceutical diet throughout the experimentation phase. HDL-c levels of hypercholesterolemic rats fed on functional and nutraceutical diets were raised up to 1.93 and $2.79 \%$ from baseline values, respectively (Table 7).

\section{Hematological aspects}

Statistical analysis demonstrated non-significant effect of diets while substantial effect of study intervals on RBC count in study I (normal rats). Further, significant effect of diets and non-significant response of study intervals was viewed in study II (hyperglycemic rats) and study III (hypercholesterolemic rats). In case of WBCs, nonsubstantial effect of diets and study intervals was noted in study I (normal rats), study II (hyperglycemic rats) and study III (hypercholesterolemic rats). For platelets, the impact of diets and study intervals was significant in case of study I (normal rats). In study II (hyperglycemic rats) and study III (hypercholesterolemic rats), the effect of diets and study intervals was non-significant on platelets (Tables 8, 9 and 10).

\section{Discussion}

Current explorations were in harmony with the findings of Coudray et al. [25] who reported that rats preferred control diet $(25.10 \mathrm{~g} /$ day) over funtional diet (19.40 to $22.20 \mathrm{~g} /$ day). In another study, Khalesi et al. [9] fed different levels of raw and heated flaxseed to Sprague

Table 9 Effect of diets and study intervals on WBC $\left(10^{3} / \mu \mathrm{L}\right)$ of rats in different studies

\begin{tabular}{|c|c|c|c|c|c|}
\hline \multirow[t]{2}{*}{ Studies } & \multirow[t]{2}{*}{ Diets } & \multicolumn{3}{|c|}{ Study intervals (Days) } & \multirow[t]{2}{*}{ Means } \\
\hline & & 0 & 28 & 56 & \\
\hline \multirow[t]{4}{*}{ Study 1} & Control & $10.56 \pm 0.13$ & $10.98 \pm 0.18$ & $11.04 \pm 0.20$ & $10.86 \pm 0.17$ \\
\hline & Functional & $10.37 \pm 0.21$ & $10.54 \pm 0.23$ & $10.74 \pm 0.18$ & $10.55 \pm 0.19$ \\
\hline & Nutraceutical & $10.67 \pm 0.12$ & $10.75 \pm 0.19$ & $10.83 \pm 0.21$ & $10.75 \pm 0.18$ \\
\hline & Means & $10.53 \pm 0.18$ & $10.76 \pm 0.20$ & $10.87 \pm 0.19$ & \\
\hline \multirow[t]{4}{*}{ Study II } & Control & $10.87 \pm 0.23$ & $10.75 \pm 0.27$ & $10.64 \pm 0.41$ & $10.75 \pm 0.35$ \\
\hline & Functional & $10.81 \pm 0.24$ & $11.14 \pm 0.35$ & $11.43 \pm 0.19$ & $11.13 \pm 0.27$ \\
\hline & Nutraceutical & $10.90 \pm 0.35$ & $11.18 \pm 0.41$ & $11.42 \pm 0.34$ & $11.17 \pm 0.38$ \\
\hline & Means & $10.86 \pm 0.25$ & $11.02 \pm 0.32$ & $11.16 \pm 0.38$ & \\
\hline \multirow[t]{4}{*}{ Study 111} & Control & $11.28 \pm 0.29$ & $11.21 \pm 0.25$ & $11.15 \pm 0.27$ & $11.21 \pm 0.26$ \\
\hline & Functional & $11.37 \pm 0.29$ & $11.42 \pm 0.34$ & $11.48 \pm 0.32$ & $11.42 \pm 0.31$ \\
\hline & Nutraceutical & $11.52 \pm 0.38$ & $11.58 \pm 0.30$ & $11.64 \pm 0.35$ & $11.58 \pm 0.34$ \\
\hline & Means & $11.39 \pm 0.34$ & $11.40 \pm 0.28$ & $11.42 \pm 0.31$ & \\
\hline
\end{tabular}


Table 10 Effect of diets and study intervals on platelets $\left(10^{3} / \mu \mathrm{L}\right)$ of rats in different studies

\begin{tabular}{|c|c|c|c|c|c|}
\hline \multirow[t]{2}{*}{ Studies } & \multirow[t]{2}{*}{ Diets } & \multicolumn{3}{|c|}{ Study intervals (Days) } & \multirow[t]{2}{*}{ Means } \\
\hline & & $\overline{0}$ & 28 & 56 & \\
\hline \multirow[t]{4}{*}{ Study 1} & Control & $1021.70 \pm 51.05$ & $1029.70 \pm 48.10$ & $1038.10 \pm 51.90$ & $1029.80 \pm 50.40^{b}$ \\
\hline & Functional & $1015.70 \pm 50.10$ & $1056.70 \pm 49.30$ & $1082.10 \pm 54.20$ & $1051.40 \pm 52.50^{\mathrm{ab}}$ \\
\hline & Nutraceutical & $1018.70 \pm 52.45$ & $1076.70 \pm 53.60$ & $1103.30 \pm 55.80$ & $1066.20 \pm 53.30^{a}$ \\
\hline & Means & $1018.70 \pm 50.90^{b}$ & $1054.30 \pm 52.70^{\mathrm{a}}$ & $1074.40 \pm 53.85^{a}$ & \\
\hline \multirow[t]{4}{*}{ Study II } & Control & $1034.30 \pm 52.20$ & $1023.00 \pm 52.65$ & $1016.30 \pm 53.10$ & $1024.53 \pm 52.80$ \\
\hline & Functional & $1037.70 \pm 53.60$ & $1046.70 \pm 51.40$ & $1059.30 \pm 51.30$ & $1042.30 \pm 52.65$ \\
\hline & Nutraceutical & $1029.70 \pm 52.05$ & $1044.70 \pm 52.80$ & $1052.70 \pm 50.05$ & $1047.90 \pm 51.40$ \\
\hline & Means & $1033.90 \pm 51.90$ & $1038.13 \pm 52.05$ & $1040.76 \pm 52.50$ & \\
\hline \multirow[t]{4}{*}{ Study 111} & Control & $1035.10 \pm 49.50$ & $1023.70 \pm 50.20$ & $1011.70 \pm 48.90$ & $1023.40 \pm 49.70$ \\
\hline & Functional & $1034.70 \pm 50.85$ & $1045.00 \pm 49.15$ & $1049.30 \pm 46.40$ & $1043.00 \pm 51.30$ \\
\hline & Nutraceutical & $1041.70 \pm 49.25$ & $1048.70 \pm 51.40$ & $1064.10 \pm 47.20$ & $1051.40 \pm 50.65$ \\
\hline & Means & $1037.16 \pm 47.65$ & $1039.13 \pm 50.95$ & $1041.70 \pm 49.10$ & \\
\hline
\end{tabular}

Study I (Normal rats), Study II (Hyperglycemic rats), Study III (Hypercholesterolemic rats). Lettering indicates significant differences between means using ANOVA (two-way factorial) with posthoc Tukey's honestly significant difference (HSD) test

Dawley rats and observed that feed consumptions was slightly lower in groups fed with higher percentage of flaxseed. Moreover, they mentioned comparatively less weight gain in rats fed on flaxseed (30\% of total diet) as compared to control animals. In the current study, nonsignificant increase in water intake was noted throughout the trial among different diet groups. Likewise, Yang et al. [26] observed non-substantial variances in water intake among functional or control diets fed groups.

Hypoglycemic prespectives of flaxseed as observed in the current research were in acordance to the outcomes of Hejazi [15]. In the mentioned study, blend of flaxseed $(50 \%)$ and hull-less barley (50\%) was administered to rats that indicated reduction in glucose (34.61\%), cholesterol (49.05\%), LDL-c (69.09\%) and triacylglycerols (55.19\%) along with improvent in HDL-c (69.13\%) in streptozotocyin induced diabetic rats. Conclusively, they found that barley and flaxseed blend is a unique combination of soluble fiber and polyunsaturated fatty acids that protect against heart disorders. Earlier, Elshal et al. [27] found flaxseed helpful in controlling glucose, cholesterol, triacylglycerol and LDL-c up to 68, 51, 52 and $66 \%$ along with increase in HDL-c by $58 \%$ as compared to positive control (diabetic rats). Moreover, Makni et al. [5] administered flaxseed and pumpkin seed mixture to diabetic rats that resulted in significant drop of plasma cholesterol and triacylglycerols up to $47 \%$, each in contrast to diabetic rats. The combination of flaxseed and pumpkin ensures the provision of phytosterols, polyunsaturated fatty acids, tocopherols and $\beta$-carotene. Previously, Haliga et al. [28] demonstrated that supplementation of flaxseed down-regulated cholesterol by $24.90 \%$ and raised serum HDL-c up to $91 \%$ in streptozotocin induced diabetic hamsters as compared to control diabetic hamsters.
Regarding lipid profile, Gambus et al. [29] found that rats administered with bread containing flaxseed (10 and 13\%) achieved impressive hypocholesterolemic effects in rats in response to higher dietary fiber and microminerals. Earlier, Khalesi et al. [9] studied the effect of 10,20 and $30 \%$ raw and heated flaxseed on the serum lipid profile of normal diet fed rats and noted that group fed on $30 \%$ flaxseed showed maximum reduction in cholesterol (22.08\%), LDL-c (4.00\%) and triacylglycerols $(7.69 \%)$ along with maximum increment in HDL-c (6.02\%) followed by $20 \%$ and $10 \%$ flaxseed groups. They associated such effects with soluble dietary fiber and SDG content in flaxseed. Further, Zhang et al. [30] showed significant decline in total cholesterol \& LDL-c upto $24.38 \%$ and fasting plasma glucose by $24.96 \%$ in hypercholesterolemic subjects, administered with $600 \mathrm{mg}$ of SDG at 8th week as compared to placebo or baseline. One of the researchers group, Jenkins et al. [31] reported that subjects supplemented with partially de-fatted flaxseed based muffins for 3 weeks helped in lowering cholesterol level by $5.5 \%$ in response to flaxseed gum i.e. a rich source of phenolic lignan however, non-significant responses were noted in lipoprotein fractions.

Hematological aspects in the current study showed non-significant results that were in line with findings of Prasad [32] who explicated that counts of red blood cells, white blood cells and platelets remained unaltered after flaxseed diet in normal and hypercholesterolemic rabbits. One of the scientists group, Babu et al. [33] also elucidated that dietary flaxseed meal had no adversities on hematological traits in Sprague-Dawley rats.

\section{Conclusions}

The flaxseed extract (5\%) based diet demonstrates better control on glucose, cholesterol, LDL-c and triacylglycerols 
as compared to flaxseed powder (10\%). On the other hand, functional and nutraceutical diets portray increment in insulin and HDL-c levels in hyperglycemic and hypercholesterolemic diet fed rats. Thus, incorporation of flaxseed in dietary regimen helps to mitigate lifestyle related dysfunctions.

\section{Abbreviations}

END: Enterodiol; ENL: Enterolactone; HDL-c: High density lipoproteincholesterol; LDL-c: Low density lipoprotein-cholesterol; RBC: Red blood cell; SDG: Secoisolariciresinol diglucoside; WBC: White blood cell

\section{Acknowledgements}

The authors are thankful to Functional and Nutraceutical Food Research Section, National Institute of Food Science and Technology, University of Agriculture, Faisalabad, Pakistan.

\section{Authors' contributions}

UA designed the project under the supervision of MSB and FA, AB \& HARS prepared the final manuscript. All authors read and approved the final manuscript.

\section{Funding}

Not applicable.

\section{Availability of data and materials}

Not applicable.

\section{Ethics approval and consent to participate}

Ethical approval was given by the head of National Institute of Food Science and Technology (NIFSAT), Faculty of Food, Nutrition \& Home Sciences, University of Agriculture Faisalabad (UAF), Pakistan by considering the suggestions of Animal Experimentation Ethics Committee, UAF. Male white Sprague Dawley rats were housed in the Animal Room of the NIFSAT, UAF by following the instructions for care provided by the committee of the university.

\section{Consent for publication}

Not applicable.

\section{Competing interests}

The authors declare that they have no competing interests.

\section{Author details}

'Department of Food Processing and Preservation Technology, Government College of Technology Sahiwal, Sahiwal, Pakistan. ${ }^{2}$ National Institute of Food Science and Technology, Faculty of Food, Nutrition \& Home Sciences, University of Agriculture Faisalabad, Faisalabad, Pakistan. ${ }^{3}$ Department of Food Science and Technology, Faculty of Science and Technology, Government College Women University Faisalabad, Faisalabad, Pakistan. ${ }^{4}$ University Institute of Diet and Nutritional Sciences, Faculty of Allied Health Sciences, The University of Lahore, Lahore, Pakistan. ${ }^{5}$ Q Diamantina Institute, Translational Research Institute, Faculty of Medicine, The University of Queensland, 37 Kent Street Woolloongabba, Brisbane, QLD 4102, Australia. ${ }^{6}$ Centre for Chemistry and Biotechnology, School of Life and Environmental Sciences, Deakin University, Pigdons Road, Waurn Ponds, VIC 3216, Australia. ${ }^{7}$ School of Agriculture and Food, The University of Melbourne, Parkville, VIC 3010, Australia. ${ }^{8}$ Department of Agriculture and Food Systems, The University of Melbourne, Level 3, 780 Elizabeth Street, Parkville, VIC 3010, Australia.

\section{Received: 3 September 2019 Accepted: 5 January 2020}

\section{6.}

\section{References}

1. Tewfik S, Tewfik I. Nutraceuticals, functional foods and botanical dietary supplements; promote wellbeing and underpin public health. World Rev Sci Technol Sust Dev. 2008;5(2):104-23.

2. Day L, Seymour RB, Pitts KF, Konczak I, Lundin L. Incorporation of functional ingredients into foods. Trends Food Sci Tech. 2009;20(9):388-95.
3. Shahidi F. Nutraceuticals and functional foods: whole versus processed foods. Trends Food Sci Technol. 2009;20:376-87.

4. Bigliardi B, Galati F. Innovation trends in the food industry: the case of functional foods. Trends Food Sci Technol. 2013:31(2):118-29.

5. Makni M, Hamadi F, Nabil K, Mouldi G, Najiba Z. Antidiabetic effect of flax and pumpkin seed mixture powder: effect on hyperlipidemia and antioxidant status in Alloxan diabetic rats. J Diabetes Complicat. 2011;25(5): 339-45.

6. Hussein SA, El-Senosi YA, Ragab MR, Hammad MM. Beneficial effect of flaxseed oil on lipid metabolism in high cholesterol diet fed rats. Banha Vet Med J. 2014;27(2):290-301.

7. Pradhan $R$, Meda $V$, Rout $P$, Naik S. Supercritical $\mathrm{CO}_{2}$ extraction of fatty oil from flaxseed and comparison with screw press expression and solvent extraction processes. J Food Eng. 2010;98(4):393-7.

8. Bozan B, Temelli F. Chemical composition and oxidative stability of flax, safflower and poppy seed and seed oils. Bioresour Technol. 2008;99(14): 6354-9.

9. Khalesi S, Jamaluddin R, Ismail A. Effect of raw and heated flaxseed (Linum Usitatissimum L.) on blood lipid profiles in rats. Int J Appl Sci Technol. 2011; 1(4):84-9.

10. Ganorkar PM, Jain RK. Flaxseed--a nutritional punch. Int Food Res J. 2013; 20(2):519-25.

11. Kajla P, Sharma A, Sood DR. Flaxseed-a potential functional food source. J Food Sci Tech. 2015;52(4):1857-71.

12. Prasad K, Jadhav A. Prevention and treatment of atherosclerosis with flaxseed-derived compound secoisolariciresinol diglucoside. Curr Pharm Des. 2016;22(2):214-20

13. Kamal-Eldin A, Peerlkamp N, Johnsson P, Andersson R, Lundgren L, Aman P. An oligomer from flaxseed composed of secosolariciresinoldiglucoside and 3-hydroxy-3-methyl glutaric acid residues. Phytochem. 2001;58(4):587-90.

14. Eliasson C, Kamal-Eldin A, Andersson R, Aman P. High performance liquid chromatographic analysis of secoisolariciresinol diglucoside and hydroxycinnamic acid glucosides in flaxseed by alkaline extraction. J Chromatogr A. 2003;1012(2):151-9.

15. Hejazi MA. Using Hull-less barley and flax seeds flour to produce macaroni for hyperglycemia disease in rats. Life Sci J. 2014;11(2):354-61.

16. Ho C, Cacace J, Mazza G. Extraction of lignans, proteins and carbohydrates from flaxseed meal with pressurized low polarity water. Lebenson Wiss Technol. 2007;40(9):1637-47.

17. Wolf BW, Weisbrode SE. Safety evaluation of an extract from Salacia oblonga. Food Chem Toxicol. 2003;41(16):867-74.

18. Kim Jl, Paik JK, Kim OY, Park HW, Lee JH, Jang Y, Lee JH. Effects of lycopene supplementation on oxidative stress and markers of endothelial function in healthy men. Atheroscl. 2011;215(1):189-95.

19. Ahn J, Choi W, Kim S, Ha T. Anti-diabetic effect of watermelon (Citrullus vulgaris schrad) on streptozotocin-induced diabetic mice. Food Sci Biotechnol. 2011:20(1):251-4.

20. Buriro MA, Tayyab M. Effect of Nigella sativa on lipid profile in albino rats. Gomal J Med Sci. 2007:5(1):28-31.

21. Alshatwi AA, Al-Obaaid MA, Al-Sedairy SA, Al-Assaf AH, Zhang JJ, Lei K. Tomato powder is more protective than lycopene supplement against lipid peroxidation in rats. J Nut Res. 2010;30(1):66-73.

22. Kamatani $Y$, Matsuda K, Okada Y, Kubo M, Hosono N, Daigo Y, Nakamura Y, Kamatani N. Genome-wide association study of hematological and biochemical traits in a Japanese population. Nat Genet. 2010:42(3):210-5.

23. Al Haj M, Kazzam E, Nagelkerke NJ, Nyberg F, Nicholls MG, Adem A. Effect of dehydration in the presence and absence of the angiotensin receptor blocker losartan on blood constituents in the camel. J Med Sci. 2011;4(2): 73-8.

24. Mason RL, Gunst RF, Hess JL. Statistical design and analysis of experiments. Hoboken: Wiley; 2003

25. Coudray C, Verney MAL, Tressol JC, Coudary CF, Molteni NMH, Demigne C, Rayssiguir $Y$, Ramsey C. Mineral supplementation of white wheat flour is necessary to maintain adequate mineral status and bone characteristics in rats. J Trace Elem Med Biol. 2001;15(2-3):131-7.

26. Yang $\mathrm{MH}$, Wang $\mathrm{CH}$, Chen $\mathrm{HL}$. Green, oolong and black tea extracts modulate lipid metabolism in hyperlipidemia rats fed high-sucrose diet. Jutr Biochem. 2001;12(1):14-20.

27. Elshal MF, Kumosani TA, Abulnaja KO. Influence of defatted flaxseed diet on insulin sensitivity, vascular permeability and lipid profile in a rat model of type 2 diabetes mellitus. J Med Plants Res. 2012;6(11):2188-93. 
28. Haliga R, Mocanu V, Oboroceanu T, Stitt PA, Luca VC. The effects of dietary flaxseed supplementation on lipid metabolism in streptozotocin-induced diabetic hamsters. Rev Med Chir Soc Med Nat lasi. 2007;111(2):472-6.

29. Gambus H, Mikulec A, Gambus F, Pisulewski P. Perespectives of linseed utilization in baking. Polish J Food Nutr Sci. 2004;13(1):21-7.

30. Zhang W, Wang X, Liu Y, Tian H, Flickinger B, Empie MW, Sun SZ. Dietary flaxseed lignan extract lowers plasma cholesterol and glucose concentrations in hypercholesterolaemic subjects. Brit J Nutr. 2008;99(6): 1301-9.

31. Jenkins DJA, Kendall CWC, Vidgen E, Agarwal S, Rao AV, Rosenberg RS, Diamandis EP, Novokmet R, Mehling CC, Perera T, Griffin LC. Health aspects of partially defatted flaxseed including effects on serum lipids oxidative measures and ex vivo androgen and progestin activity: a controlled crossover trial. Am J Clin Nutr. 1999;69(3):395-402.

32. Prasad K. Effect of chronic administration of lignan complex isolated from flaxseed on the hemopoietic system. Mol Cell Biochem. 2005;270(1-2):139-45.

33. Babu US, Geraldine VM, Wiesenfeld P, Jenkins MY, Gowda H. Nutritional and hematological impact of dietary flaxseed and defatted flaxseed meal in rats. Int J Food Sci Nutr. 2000;51(2):109-17.

\section{Publisher's Note}

Springer Nature remains neutral with regard to jurisdictional claims in published maps and institutional affiliations.

\section{Submit your manuscript to a SpringerOpen ${ }^{\circ}$ journal and benefit from:}

- Convenient online submission

- Rigorous peer review

- Open access: articles freely available online

- High visibility within the field

- Retaining the copyright to your article

Submit your next manuscript at $\boldsymbol{\nabla}$ springeropen.com 